\title{
Thermal architecture of the ESA ARIEL payload
}

G. Morgante, L. Terenzi, D. D'Ascanio, P. Eccleston, M. Crook, et al.

G. Morgante, L. Terenzi, D. D'Ascanio, P. Eccleston, M. Crook, T. Hunt, V. Da Deppo, M. Focardi, G. Malaguti, G. Micela, E. Pace, G. Tinetti, "Thermal architecture of the ESA ARIEL payload," Proc. SPIE 10698, Space Telescopes and Instrumentation 2018: Optical, Infrared, and Millimeter Wave, 106984H (6 July 2018); doi: 10.1117/12.2313153

Event: SPIE Astronomical Telescopes + Instrumentation, 2018, Austin, Texas, United States 


\title{
Thermal architecture of the ESA ARIEL payload
}

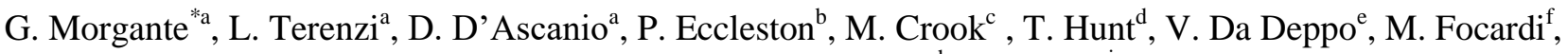 \\ G. Malaguti ${ }^{\mathrm{a}}$, G. Micela ${ }^{\mathrm{g}}$, E. Pace $^{\mathrm{h}}, \mathrm{G}^{\mathrm{a}}$. Tinetti ${ }^{\mathrm{i}}$ \\ ${ }^{a}$ INAF - OAS Bologna, via P. Gobetti, 93/3, 40129, Bologna, Italy; ${ }^{b}$ RAL Space, STFC Rutherford Appleton \\ Laboratory, Harwell Campus, Didcot OX11 0QX, UK; ${ }^{\mathrm{c}}$ Technology Department, STFC Rutherford Appleton \\ Laboratory, Harwell Campus, Didcot OX11 0QX, UK; ${ }^{\mathrm{d}}$ Mullard Space Science Laboratory, Holmbury St. \\ Mary, Dorking, Surrey RH5 6NT, UK; ${ }^{\mathrm{e}}$ IFN-CNR via Trasea 7, 35131 - Padova, Italy; ${ }^{\mathrm{f}}$ INAF - Osservatorio \\ Astrofisico di Arcetri, Largo E.Fermi 5 I-51025 Firenze, Italy; ${ }^{\mathrm{g}}$ INAF - Osservatorio Astronomico di \\ Palermo, Piazza del Parlamento 1, 90134, Palermo, Italy; ${ }^{\text {h }}$ Dip. di Fisica ed Astronomia, Università di \\ Firenze, Via Sansone, 1, 50019 Sesto Fiorentino (FI), Firenze, Italy; ${ }^{i}$ Dept. Physics and Astronomy, \\ University College London, London WC1E 6BT, UK
}

\begin{abstract}
The Atmospheric Remote-sensing Infrared Exoplanets Large-survey (ARIEL) is a space project selected by the European Space Agency as the next M4 mission within the Cosmic Vision 2015-2025 programme. ARIEL will probe the chemical and physical properties of a large number of known exoplanets by observing spectroscopically their atmosphere, to extend our knowledge of how planetary systems form and evolve. To achieve its scientific objectives, the mission is designed as a dedicated 3.5-years survey for transit and eclipse spectroscopy, with an instrumental layout based on a 1-m class telescope feeding two spectrometer channels that cover the band 1.95 to $7.8 \mu \mathrm{m}$ and four photometric channels in the visible to near-IR range.

The high sensitivity requirements of the mission need an extremely stable thermo-mechanical platform. In this paper we describe the thermal architecture of the payload and discuss the main requirements that drive the design. The ARIEL thermal configuration is based on a passive and active cooling approach. Passive cooling is achieved by a V-Groove based design that exploits the L2 orbit favorable thermal conditions. The telescope and the optical bench are passively cooled to a temperature close to $50 \mathrm{~K}$ to achieve the required sensitivity and stability. The photometric detectors are maintained at the operating temperature of $50 \mathrm{~K}$ by a dedicated radiator coupled to cold space. The IR spectroscopic channel detectors require a lower temperature reference. This colder stage is provided by an active cooling system based on a Neon Joule-Thomson cold end, fed by a mechanical compressor, able to reach temperatures lower than 30K.

Thermal stability of the telescope and detector units is one of the main drivers of the design. The periodical perturbations due to orbital changes, to the active cooling or to other internal instabilities make the temperature control one of the most critical issues of the whole architecture. The thermal control system design, based on a combination of passive and active solutions aimed at maintaining the required stability at the telescope and detector stages level, is described.

We report here about the baseline thermal architecture at the end of the Phase A, together with the main trade-offs needed to enable the ARIEL exciting science in a technically feasible payload design. Thermal modeling results and preliminary performance predictions in terms of steady state and transient behavior are also discussed.
\end{abstract}

This paper is presented on behalf of the ARIEL Consortium.

Keywords: Space instrumentation, thermal control, cryogenics, infrared, spectroscopy, exoplanets

\section{INTRODUCTION}

The Atmospheric Remote-sensing Infrared Exoplanets Large-survey (ARIEL) ${ }^{1}$ is a space project selected by the European Space Agency as the next medium-size mission (M4) within the Cosmic Vision 2015-2025 programme.

Observations of the increasing number of exoplanets by space and ground telescopes show an incredible variety of characteristics (orbits, dimensions, temperature, composition, atmosphere, etc.) indicating that planetary systems appear much more diverse than expected.ARIEL ${ }^{1}$ is the first dedicated mission to investigate exoplanetary atmospheres and to

Space Telescopes and Instrumentation 2018: Optical, Infrared, and Millimeter Wave, edited by Makenzie Lystrup,

Howard A. MacEwen, Giovanni G. Fazio, Proc. of SPIE Vol. 10698, 106984H

(C) 2018 SPIE · CCC code: $0277-786 X / 18 / \$ 18 \cdot$ doi: $10.1117 / 12.2313153$ 
take up the challenge to explain this diversity, providing fundamental information on formation, evolution, internal structure and planet and atmospheric composition at a level never previously achieved.

The mission is aimed at undertaking spectroscopy of transiting exoplanets over a wide wavelength range, providing high resolution, multi-wavelength spectroscopic observations on the atmospheres of a large and representative selected sample of known warm and hot exoplanets. ARIEL results could constrain models of the planets internal structure and improve our understanding of how they form and evolve, addressing their suitability for life and placing our Solar System in context.

ARIEL $^{2}$ will carry a single cryogenic, high stability spectrometer covering the IR band 1.95-7.80 $\mu \mathrm{m}$ with a resolving power of about 200 mounted on a single optical bench with the telescope and a Fine Guidance Sensor (FGS) that provides closed-loop feedback to the high stability pointing of the spacecraft. The FGS provides also simultaneous information on the photometric stability of the target stars. The instrument channels are fed by a 1-m class afocal telescope, composed of an off-axis portion of a two-mirror classic Cassegrain coupled to a tertiary off-axis paraboloidal mirror. The optical configuration is complemented by a common optics system shared by all the instrument modules.

The ARIEL mission is complementary to other international facilities such as TESS and will build on the success of ESA exoplanet missions such as Cheops and PLATO, expected to provide an optimized target list prior to launch.

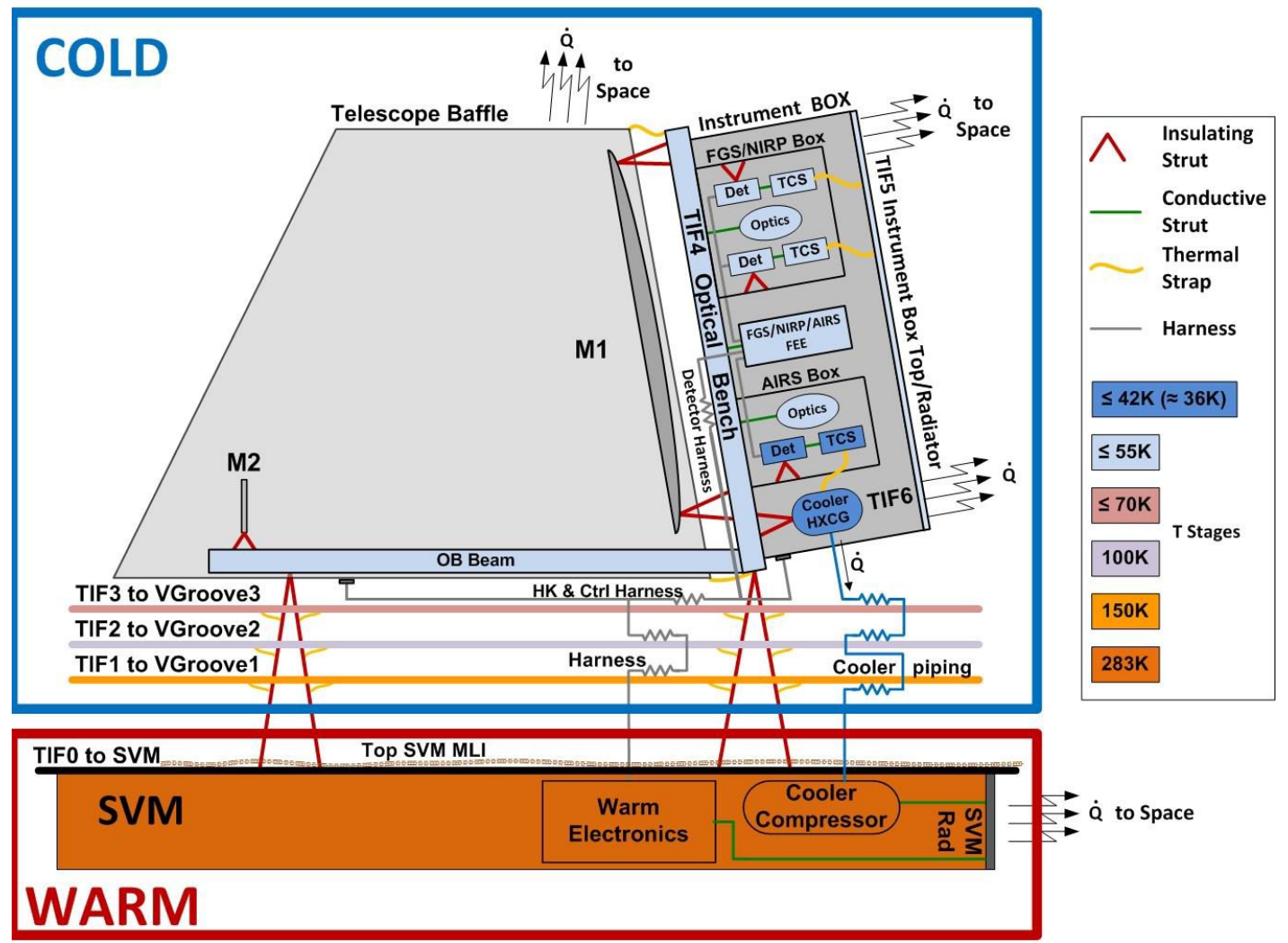

Figure 1. Block diagram of the ARIEL spacecraft

\section{PAYLOAD BASELINE THERMAL ARCHITECTURE}

To tackle its ambitious scientific program, ARIEL is designed as a dedicated survey mission for transit and eclipse spectroscopy capable of observing a large warm and hot planet sample within its 3.5-year mission lifetime ${ }^{3}$. ARIEL will be launched on board the Ariane VI launcher from the Centre Spatial Guyanais in Kourou (French Guiana), into a direct transfer leading to a large amplitude orbit around the Sun-Earth L2 point. This science operations orbit is key to meeting two of the most important requirements: it offers a very stable environment (for thermal, power and communication purposes), combined with a very large instantaneous field of regard. 
The spacecraft thermal design (Figure 1) is based on a cold Payload Module (PLM) sitting on the top of a warm Service Module (SVM). The mission thermal control is accomplished by a combination of passive and active cooling systems. The SVM is thermally controlled in the $270 \mathrm{~K}-290 \mathrm{~K}$ range for nominal operations of all the S/C subsystem units. The function of the cold PLM is to shield the scientific instrumentation (the ARIEL instrument and the telescope system) from the warm section of the S/C and to provide it with the required cooling and thermal stability.

The SVM upper surface, the main thermo-mechanical interface of the PLM to the S/C, is covered with a low emissivity MLI shroud and acts as the first main radiative barrier between the PLM and the warm units in the service module. The geometrical configuration of the PLM passive stages and the maximum Solar Aspect Angles allowed during the mission are strongly related. The SVM interface is assumed, at this stage of the study, as a perfect Sun shield for the PLM in the thermal analysis. This assumption will be verified in the next thermal analysis and design steps as the first Sun shielding stage and the PLM passive cooling system must be mutually designed. At 1.5 million km from the Earth in the anti-Sun direction, the L2 orbit allows to maintain the same spacecraft attitude relative to the Sun-Earth system, while scanning the whole sky during the mission duration. Limiting the allowed Solar Aspect Angle (SAA) range, ARIEL will operate in a very stable thermal environment keeping always protect from the Sun/Earth/Moon illumination the coldest section of the PLM. For this reason the SAA allowed during nominal observations will be limited to $\pm 6^{\circ}$ around the S/C X-axis and to $\pm 30^{\circ}$ around the $\mathrm{Y}$-axis (Figure 3).

Passive cooling is achieved by a high efficiency thermal shielding system (Figure 1 and Figure 2) based on a three VGrooves combination that, in the L2 environment, can provide stable temperature stages down to the $50-60 \mathrm{~K}$ range. They represent the first cooling stage of the PLM. Mechanically supported on the SVM by bipods and other insulating struts, their shape, geometrical configuration and optical properties allow an efficient rejection of heat to cold space (Figure 3). Past missions (such as Planck ${ }^{4}$, Spitzer Telescope and Herschel) have demonstrated that, in an environment such as L2, is possible to passively reach and maintain temperatures down to the 50K range with loads up to more than $1 \mathrm{~W}$. These three radiators, called V-Groove 1, 2 and 3 (VG1, VG2, VG3), work in sequence providing stable temperature references for the Instrument units, for parasitic heat leaks (harness, struts, radiation) interception and for cryo-cooler pre-cooling. The last V-Groove, VG3, defines the coldest passive environment of the PLM and accommodates the Instrument modules and the Telescope Assembly (Figure 2).

The telescope is required to operate at a temperature $<70 \mathrm{~K}$. The Telescope Assembly (TA), enclosed in the cold environment established by the last V-Groove, acts as an extra passive stage using its large Baffle and Optical Bench (TOB) as radiating surfaces. These radiators, with the same high emissivity coating configuration, greatly improve the efficiency and the performances of the PLM passive cooling.
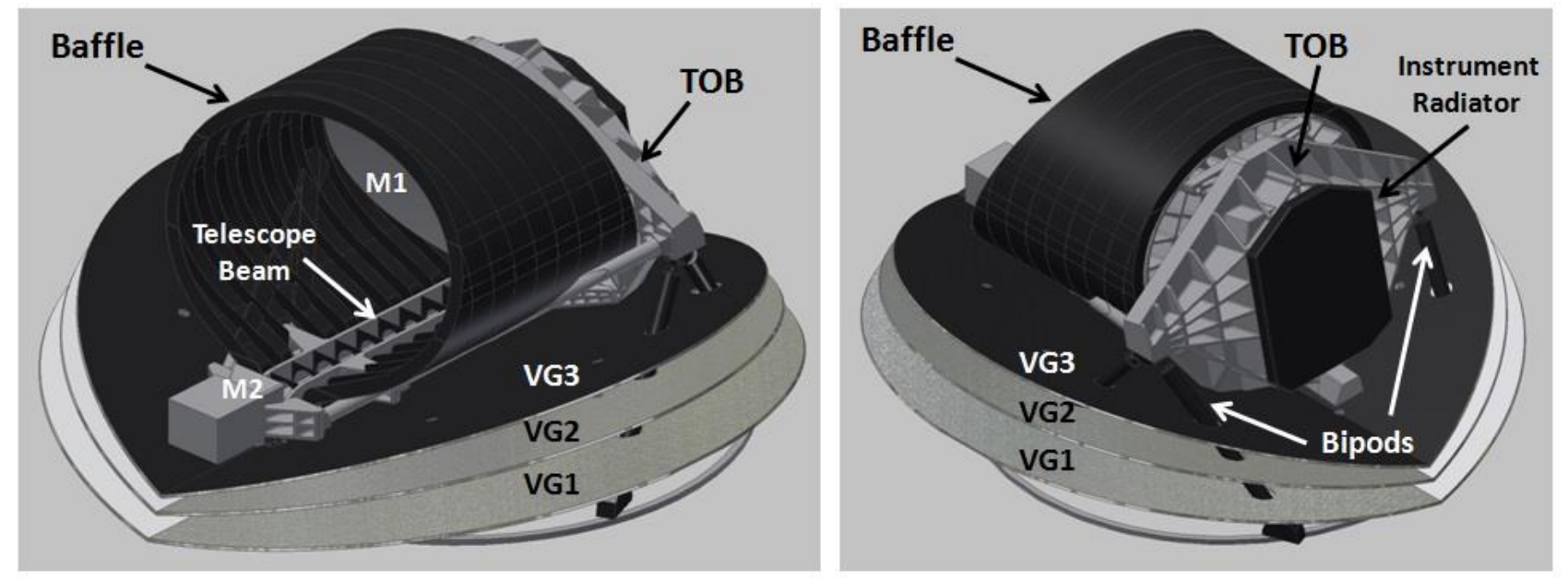

Figure 2: ARIEL PLM thermo-mechanical architecture

The ARIEL Instrument modules are integrated on the Optical Bench and their cooling is achieved in two different ways, following the temperature requirements of each frequency band. The detectors of two channels, the Fine Guidance System (FGS) and the Near Infrared Photometer (NIRP), located in a single module box (the FGS) are passively cooled to $\mathrm{T} \leq 70 \mathrm{~K}$ by a dedicated radiator represented by the top surface that closes the modules cavity on the Optical Bench. This radiator, fully enclosed in the cold radiative environment set by the last V-Groove, always faces the cold space 
during operations. The ARIEL Infra-Red Spectrometer (AIRS) detectors must be operated colder, below 42K (see Table 1 ), with the goal of approaching a temperature of $36 \mathrm{~K}$, to minimize detector noise. Maintaining this temperature, with a load of tens of $\mathrm{mW}$, require the implementation of an active cooling system. The cryocooler baseline relies on the Planck mission and $\mathrm{EChO}^{5}$ study heritage: a JT cold end fed by a Planck-like mechanical compressor using Neon gas isenthalpic expansion to achieve the required low temperature and heat lift. The design and implementation of the ARIEL active cooling system is under the responsibility of the Rutherford Appleton Laboratory (RAL) in the UK.

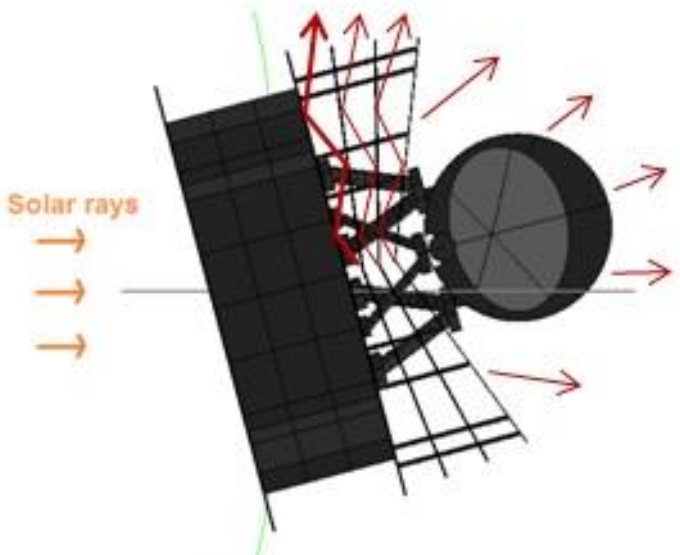

$\pm 6^{\circ}$ (X axis rotation)

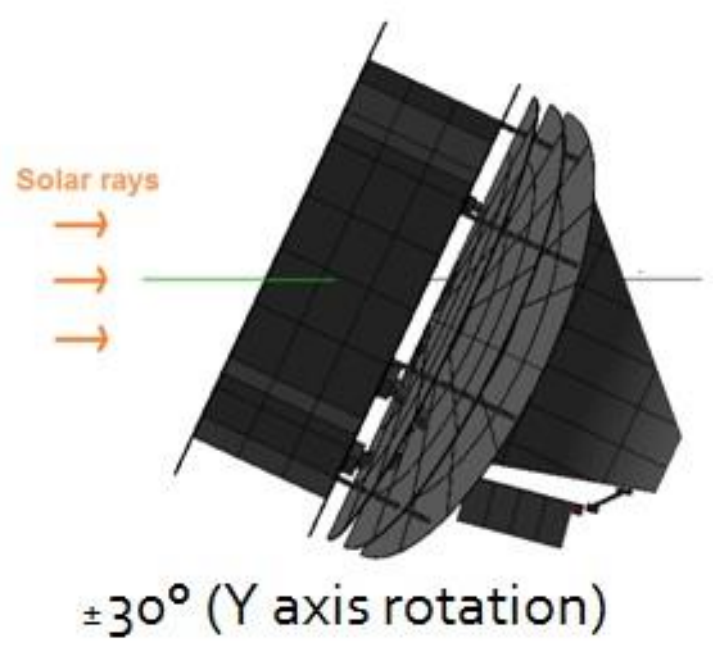

$\pm 30^{\circ}$ ( $Y$ axis rotation)

Figure 3: ARIEL S/C attitude and SAA in orbit

The general scheme of the PLM, shown in Figure 1, indicates the six main thermal interfaces of the PLM identified in the analysis. The design of the PLM thermal architecture is driven by the required operating temperature of each unit and interface, in combination with the expected loads at the thermal stages. The present assumptions for these values are reported in the following Table.

Table 1: Main thermal requirements and expected loads for the ARIEL payload

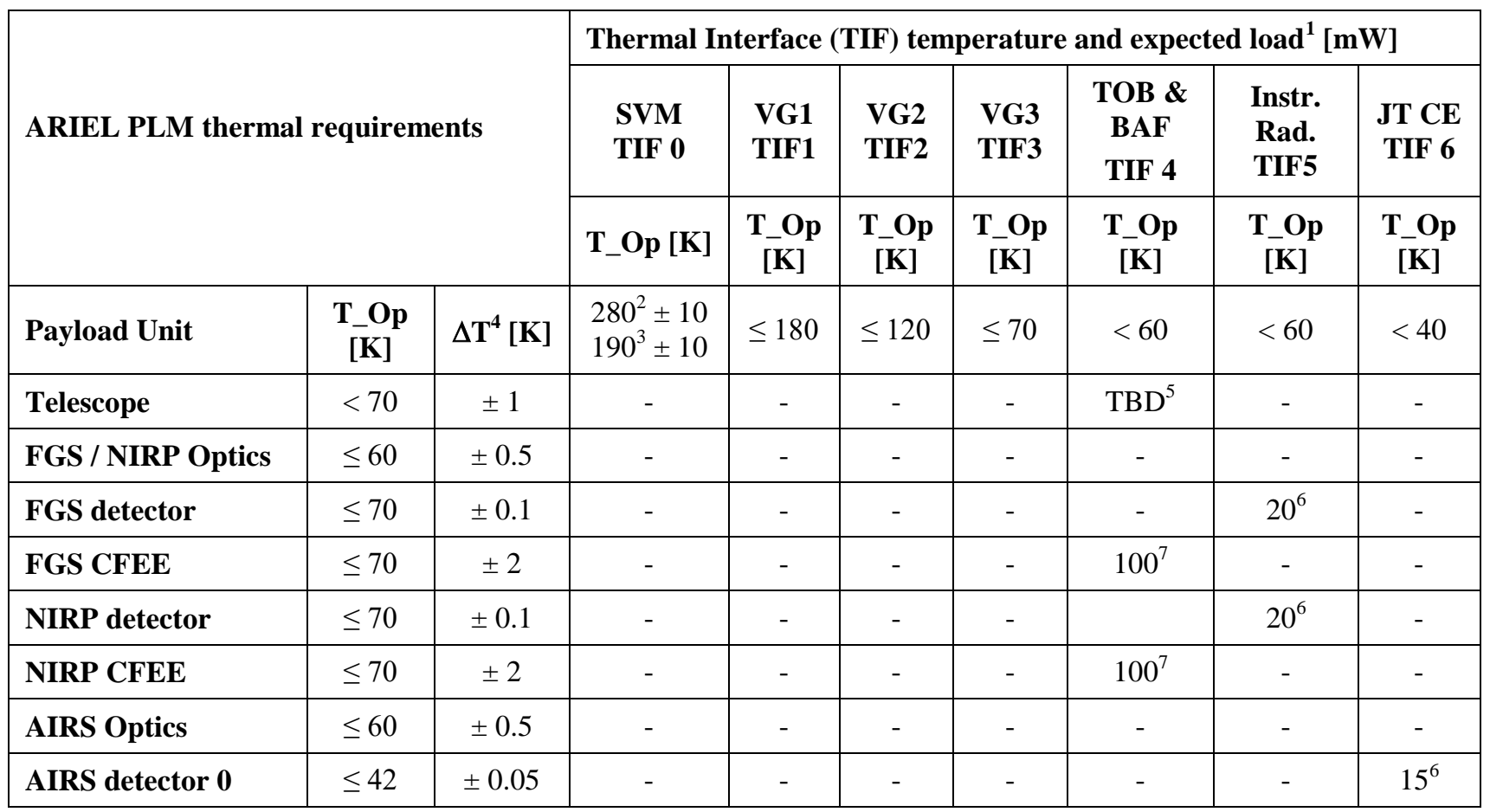




\begin{tabular}{|l|c|c|c|c|c|c|c|c|c|} 
AIRS detector 1 & $\leq 42$ & \pm 0.05 & & & & & $100^{7}$ & & $15^{6}$ \\
\hline AIRS CFEE & $\leq 62$ & \pm 2 & - & - & - & - & $100^{7}$ & - & - \\
\hline $\begin{array}{l}\text { Parasitic leaks } \\
\text { (struts + harness + } \\
\text { piping + radiation) }\end{array}$ & NA & NA & TBD $^{8}$ & TBD $^{8}$ & $\mathrm{TBD}^{8}$ & $\mathrm{TBD}^{8}$ & 50 & 10 & 5 \\
\hline
\end{tabular}

Notes: ${ }^{\prime}$ Based on assumptions and thermal analysis of present PLM design

${ }^{2}$ Conductive interface to SVM

${ }^{3}$ Radiative interface to $S V M$

${ }^{4}$ Peak to peak value over a typical observation time (7 hours)

${ }^{5} \mathrm{M} 1$ thermal control system dissipation, if needed, is expected to be $<2 \mathrm{~W}$

${ }^{6}$ FPA loads estimated with margin. The AIRS numbers already include the contribution of the FPA support and harness; $\sim 5 \mathrm{~mW}$ are the allocated for temperature control

${ }^{7}$ Worst Case dissipation

${ }^{8}$ To be evaluated by thermal analysis (see Chapter 3.2)

${ }^{9}$ Radiator with $A \geq 0.3 \mathrm{~m}^{2}$ radiator

The detectors and front-end electronics load reported in table is evaluated on the basis of the present design trade-off study of the channels detecting chain. The control stages power is a maximum average allocation for the predicted dissipation of the closed loop circuit when assumptions on the expected instabilities at the relevant thermal interfaces are made.

Besides the third V-Groove, the two critical radiators for the PLM performances are the Instrument Cavity cover and the Telescope Baffle. The baseline thermo-optical design of the external surfaces of these radiators is based on a black or white paint to maximize IR emissivity $(\varepsilon \geq 0.9)$. Instrument radiative thermal control is achieved by properly selecting the thermo-optical properties of the exposed surfaces. The radiative environment for the modules is set by the Optical Bench cavity and the Instrument Radiator that shield the channel modules and the common optics from the external environment. The remaining surface of the $\mathrm{OB}$, directly exposed to cold space, is coated with a high emissivity paint. The internal surface of the bench cavity that accommodates the instrument modules and optics requires a black coating to minimize optical stray-light leaks. For the same reason, the module boxes are assumed to be externally treated with the same black coating.

The FGS and the AIRS modules share a similar thermo-mechanical accommodation (see Figure 1). Both channels are integrated in a box that includes optical elements and a detector assembly, composed by the Focal Plane Assembly (FPA) and the cold front end electronics (CFEE). Due to electrical performance issues the cryo-harness connecting the Cold Front End Electronics (CFEE) to the detectors, the cold electronics shall be mounted on each module box in proximity of the detectors. By tuning the CFEE's thermal coupling to the OB it could be possible, if needed for performances optimization, to use their internal dissipation to keep them at a higher temperature. CFEE loads are finally rejected to space by the instrument radiator.

The FGS, that includes a near-IR photometric channel (NIRP), is integrated inside a module box with the optical units and the detectors at a temperature, respectively, $\leq 60 \mathrm{~K}$ and $\leq 70 \mathrm{~K}$. The detectors are cooled by a dedicated passive radiator stage, the Instrument Box top face (TIF5 in Figure 1) located inside the cold environment set by the third VGroove and the Telescope Assembly. This radiator is mechanically supported on the Instrument Box by means of insulating supports. The FGS/NIRP detectors are thermally decoupled from the module box and connected through high conductive links to this radiator. The FGS/NIRP box with the internal optical units is thermo-mechanically coupled to the bench by means of a conductive interface.

The AIRS detectors technology baseline requires lower operating temperatures, on the order of $40 \mathrm{~K}$ ( $\mathrm{T} \leq 42 \mathrm{~K}$ with a goal of 36K), to achieve the required sensitivity. This temperature is reached, as anticipated above, by using the Ne JT cooler. The AIRS module optics shall operate at the same temperature of the Optical Bench (60K or below). For this 
reason, while the AIRS Module Box is thermally coupled to the OB, the FPA needs to be insulated from the box, to limit the heat leak to the JT cooler cold end. In order to provide the required cooling to the AIRS detectors, the cold end heat exchanger, supported by highly insulating struts on the Optical Bench, is located within close proximity to the FPA, to minimize the thermal link length.

In general, each detector stage is thermally decoupled from the relative module box, to maximize performances of the FPA in terms of absolute temperature and stability. Coupling to the temperature reference stage is achieved through a Thermal Control Stage (TCS): this is an active closed loop thermal control system (PID type) composed by a heater + thermometer couple driven by the ICU/TCU and located on the SCA's frame. The thermal control stage of each detector is then directly coupled to the relative reference temperature stage (JT cold end or instrument radiator) by means of high conductivity thermal straps made by high purity Aluminum.

The warm electronics ${ }^{6}$ and cooler compressor are located in the SVM. All harness and piping from SVM to Instrument channels should be thermally linked to all passive stages (VG1, VG2, VG3) to minimize parasitic leaks on the instrument cooling stages. In particular, cryo-harness heat leaks to detectors shall be controlled by thermally optimizing the cables design with respect to the required electrical performances.

\section{THE ARIEL PLM THERMAL MATHEMATICAL MODEL}

The ARIEL thermal architecture development is based on the main ESATAN - TMS model of the PLM used to carry out the thermal simulations in order to verify the performances of the payload and its units and to tune the design solutions. The geometrical model (GMM) is based on the CAD configuration of the PLM. In the model the main radiative surfaces and representative supporting structures between the different stages are simulated. The conductive and radiative couplings have been assumed on the basis of the experience built with previous missions and instruments (such as Herschel/Planck, JWST MIRI and others), and verified by thermal analysis. Figure 4 shows front views of the entire model in ESATAN-TMS.

\section{SVM/PLM Interface}

In order to simulate the interaction with the Service Module, the model represents the SVM/PLM interface as a boundary reference composed by two surfaces. The conductive one is called "SVM top plate", operates at room temperature and is the main interface of the bipods and V-Grooves struts. The other surface is the "SVM Radiative shield", located in between the SVM top plate and the VG1, and represents a purely radiative coupling with the rest of the model. The SVM top plate is colored in orange, the SVM Radiative shield in yellow. These two shells have assigned optical coating MLI which has a low value of emissivity (0.05).
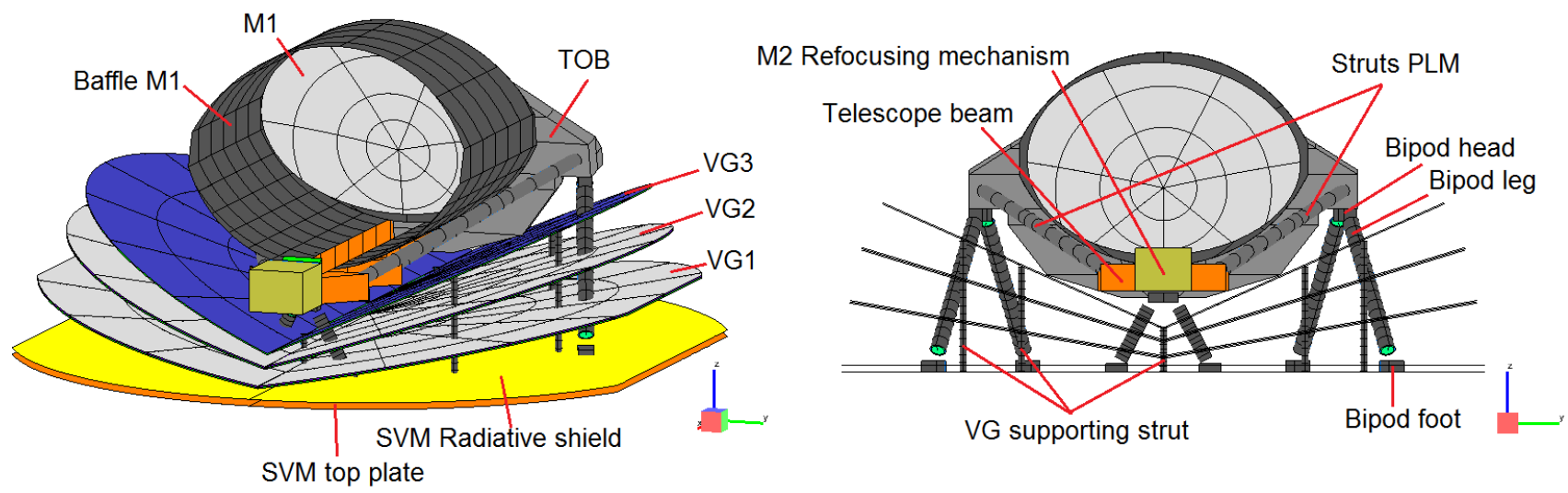

Figure 4: Front views of the PLM G/TMM with the SVM/PLM I/F. 


\section{V-Grooves, bipods and struts}

The PLM first cooling stage is represented by the V-Grooves (VGs), which are designed as three couples of semicircular panels with an inclination set of $10^{\circ}-17^{\circ}-24^{\circ}$. The V-Grooves structure, light but rigid, is composed of an $\mathrm{Al}$ sandwich with an internal honeycomb structure and Al 6061 external surfaces. The thermo-optical efficiency of this radiators system relies on angled highly-reflective surfaces open to space, rejecting radiation after a number of reflections between the angled shields (see Figure 3). Only the upper surface of the third radiator (V-Groove 3), always exposed to deep space during operations, is coated with a high IR emissivity layer (black or white paint) to maximize heat rejection.

The entire PLM passive cooling works only if the spacecraft cold section is shaded from the Sun once in orbit. The maximum allowed Solar Aspect Angle (SAA), with respect to the nominal attitude (Sun vector perpendicular to the SVM platform) is $\pm 30^{\circ}$ along the $\pm \mathrm{Y}_{\mathrm{ARIEL}}$ axis and $\pm 6^{\circ}$ along the $\pm \mathrm{X}_{\mathrm{ARIEL}}$ axis. This requirement, together with the dimensions of the SVM/PLM I/F, poses a limit on the maximum dimensions of the VGs. The radii of the designed VG's are $1260,1180,1150 \mathrm{~mm}$, respectively from the lowest to topmost. In order to have all three VGs inside the allowed envelope, it is necessary to cut the lowest VG (VG1) at the $\pm Y_{\text {ARIEL }}$ ends with a plane inclined of $6^{\circ}$ from the SVM top plate. The other two VGs (central and topmost) fit already inside the shadowed envelope.

Three bipods and eight supporting struts connect the VG's, the OB and the telescope structure to the SVM top platform (Figure 4). The bipods support the telescope structure and the Optical Bench (TOB). The struts are needed to support the V-Groove petals. Pod legs and supporting struts are made of GFRP and are modelled with hollow cylinder geometries. The bipods feet and heads are represented as Ti6Al4V fixtures, thermally linked to the legs with a calculated conductance. The legs are filled with a special rigid foam to enhance their structural properties. Dedicated links (user defined conductors) are evaluated to model the straps that thermally connect them to the V-Grooves.

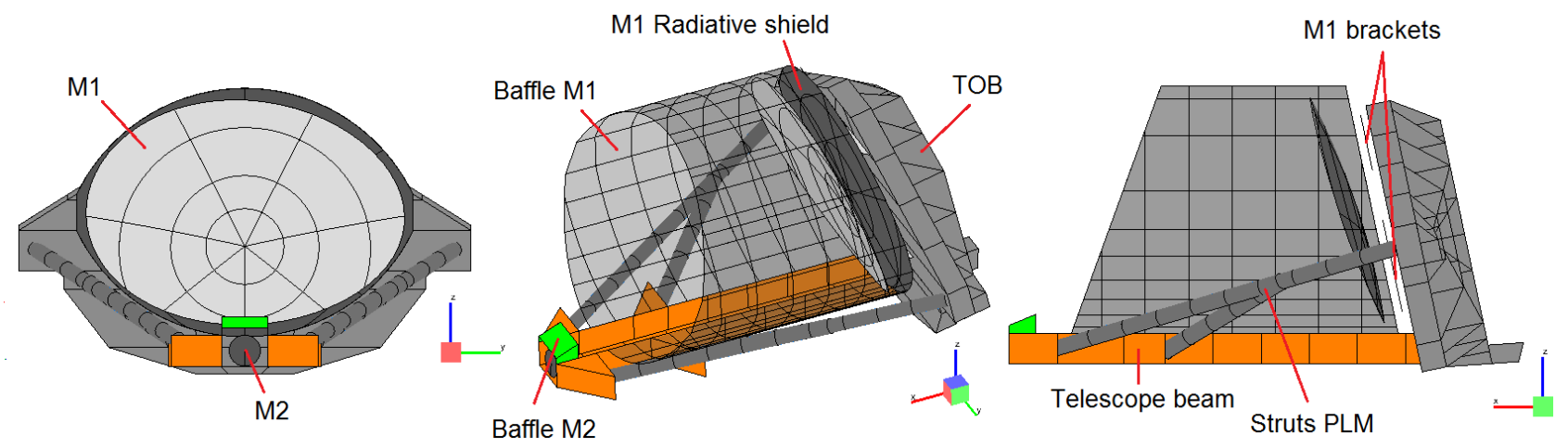

Figure 5: Three views of the Baffle, Telescope, TOB assembly. The Baffle is transparent to show the internal components.

\section{Telescope Assembly}

The Telescope Assembly (TA) is considered composed by the structure (beam), the baffle, the OB and the M1 and M2 mirrors. The $\mathrm{M}^{7}$ mirror $(1100 \mathrm{~mm} \times 750 \mathrm{~mm})$ is simulated by a cut paraboloid shape while $\mathrm{M} 2$ is a simple disc shell. The bulk material of the mirrors is $\mathrm{Al} 6061$ and their thermo-optical configuration is based on a very low emissivity front surface, while the back is assumed black painted to maximize passive cooling. In order to take into account the lightweighting cavities of the M1 back structure, an effective thickness is applied to the M1 shell geometry to replicate the expected mass.

The Telescope Baffle structure operates as an optical shield and as a thermal radiator at the same time. Exploiting its large surface, inside the radiative environment set by the last V-Groove, it is possible to enhance significantly the heat rejection of the PLM coldest part to space. For these reasons, the external and internal surfaces of the baffle are black painted in order to have a high efficient radiative exchange to cold environment and to minimize stay-light reflections in the inner volume of the telescope assembly.

The secondary mirror (M2), with its refocusing mechanism and baffle, is placed at the front aperture of the Baffle and is connected to the OB by the telescope beam. This beam, made of black painted Al 6061, supports the TOB by two Al stiffening arms used to increase the rigidity of the whole Telescope Assembly. 


\section{Instrument assembly}

On the back of the telescope assembly, the TOB accommodates in a cavity (see Figure 6) the two channel modules, FGS and AIRS, with the Cold Front End Electronics (CFEE) located on the external wall of each box adjacent to the detectors position. Each instrument is modelled as an Al 6061 box, black painted, containing the detectors. The AIRS module box contains also the active JT cooler cold end interface which is conductively linked to the two AIRS detectors.

The TOB cavity, with the modules, is closed by the Instrument Radiator that works as the cold temperature reference for the FGS/NIRP detectors. Its thermo-optical configuration is based on the same high IR emissivity coating of the Baffle to which the radiator is also linked by high thermal conductance straps.

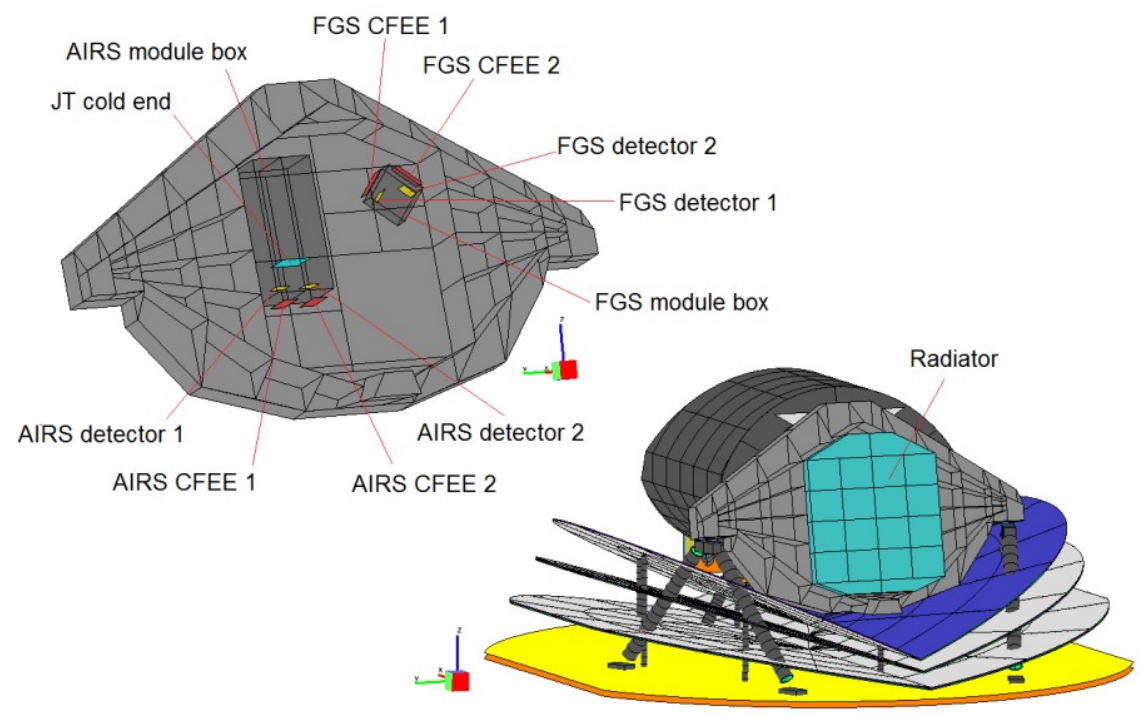

Figure 6: The upper left panel is a detailed view, through transparent module boxes, of the components inside the TOB (the instrument radiator is hidden). The CFEE are coloured in red, the detectors in yellow, the JT cold end in cyan. In the bottom-right panel a rear view of the PLM, with all geometries visible, is shown.

\subsection{Thermal Model Results}

In order to fully characterize the PLM system thermal performance in the nominal steady-state thermal conditions, a Hot and Cold case have been analysed. Table 1 reports the power boundary conditions considered for the nominal operation cases. The table includes the power dissipation of the instrument detectors and the CFEEs and the heat lift of the JT cold end assumed in the thermal model. In order to simulate the impact of the active cryo-cooler precooling load on the PLM thermal balance, a load of 120 and $360 \mathrm{~mW}$ has been applied on, respectively, VG2 and VG3. These loads simulate the power intercepted by the V-Grooves for pre-cooling the working fluid of the JT active cooler (as per RAL estimation).

The JT cooler heat exchanger is, at this stage, simulated in two different ways:

a. as a diffusion node with a fixed heat lift (negative power)

b. as a boundary (perfect heat sink) at $28 \mathrm{~K}$

In the first case no assumption was made on two-phase characteristics of the internal fluid flow. Once a cooling power map and two-phase flow properties will be available a more realistic simulation of the JT heat exchanger will be implemented. Even if the baseline cooler for ARIEL can be sized to be capable of producing a cooling power up to 50 $\mathrm{mW}$ or more, for thermal analysis purposes an extra margin of $25 \%$ has been assumed on this number. In the present thermal design of the PLM, the JT cold tip balance temperature reaches the $35-36 \mathrm{~K}$ range with $37.5 \mathrm{~mW}$ of heat lift produced. The second case, with the JT heat exchanger as a boundary node at $28 \mathrm{~K}$, is used to evaluate the resulting dissipation and compare it to the heat lift capacity of the baseline cooler $(50 \mathrm{~mW})$. In both cases a user defined conductor is used in the TMM to simulate the highly insulating strut supporting the cold end on the TOB. 
The AIRS SCA's active load assumed for the thermal analysis (Table 1) is $15 \mathrm{~mW}$ (with margin) but that number already includes the parasitic leaks (struts, harness) to the FPA that are the dominant contribution over the actual electrical dissipation. The TMM computes independently the heat leaks due to the struts (the main parasitic load $\sim 6 \mathrm{~mW}$ ). For this reason in the TMM the dissipation associated to each AIRS SCA's is $10 \mathrm{~mW}$, which corresponds to an extra $35 \%$ margin assumed over the actual active load of the detectors stage.

The radiative cases define how the model interacts with the environment. For the purpose of this paper we report here only the nominal operations orbital case, with the PLM orbiting around the Earth-Sun L2 point. In this scenario, the PLM is placed at a circular Sun-centered orbit at $1.5 \times 10^{6} \mathrm{~km}$ from the Sun, with the SVM plate perpendicular to the Sun vector. The Sun illuminates the lower surface of the SVM top plate which is assumed to totally shield the PLM from solar radiation. As already mentioned, in the present model assumptions the SVM top plate is simulated as a boundary node with a fixed temperature condition. Given the assumed radiative configuration, the PLM always results fully shaded from the Sun as far as the S/C attitude rotation remains within the allowed SAA ( $\pm 6^{\circ} \mathrm{X}_{\mathrm{ARIEL}}, \pm 30^{\circ} \mathrm{Y}_{\mathrm{ARIEL}}$ ). For this reason no radiative case with varying SAA is shown here.

Table 2 reports the solved analysis cases with the associated radiative case and boundary conditions.

Table 2: Nominal analysis cases

\begin{tabular}{|c|c|c|}
\hline Analysis case & Radiative case & Boundary conditions \\
\hline $\begin{array}{l}\text { Cold case } \\
\text { Nominal } \\
\text { Operations }\end{array}$ & $\begin{array}{l}\text { Earth-Sun } \\
\text { L2 point }\end{array}$ & $\begin{array}{l}\text { - SVM top plate @ 270K (fixed) } \\
\text { - SVM Radiative shield @ 180K (fixed) } \\
\text { - Loads in Table } 1 \\
\text { - Case 1: JT cold end as diffusion node with negative load }(-37.5 \mathrm{~mW} \text { ) } \\
\text { - Case 2: JT cold end as fixed boundary at } 28 \mathrm{~K}\end{array}$ \\
\hline $\begin{array}{l}\text { Hot case } \\
\text { Nominal } \\
\text { Operations }\end{array}$ & $\begin{array}{l}\text { Earth-Sun } \\
\text { L2 point }\end{array}$ & $\begin{array}{l}\text { - SVM top plate @ 290K (fixed) } \\
\text { - SVM Radiative shield @ 200K (fixed) } \\
\text { - Loads in Table } 1 \\
\text { - Case 1: JT cold end as diffusion node with negative load }(-37.5 \mathrm{~mW} \text { ) } \\
\text { - Case 2: JT cold end as fixed boundary at 28K }\end{array}$ \\
\hline
\end{tabular}

Table 3 shows the solved steady-state temperatures of the Cold and Hot case for the nominal conditions, corresponding to the S/C orbiting at the Earth-Sun L2 point in routine operations attitude and loads conditions. The Cold and Hot Cases are different just for the fixed boundary temperatures applied at the SVM/PLM I/F. In the table, the temperature results for both cases of the JT cold end configuration (diffusion node with fixed heat lift and boundary at 28K) are compared to the requirements with the margins to be assumed at this stage of the design process. The temperature of all pas sively and actively cooled units are fully compliant to the requirements including margins. As expected, the only relevant difference between the two configurations of the JT cold end is related to the AIRS detectors temperature: in the case of the fixed boundary at $28 \mathrm{~K}$, the AIRS SCAs result $8.5 \mathrm{~K}$ lower and well below the T requirements including margin. All other temperature values result within $0.1 \mathrm{~K}$ in both cold end simulation cases.

Table 3. Steady-state temperatures for the cold and hot case in nominal conditions.

\begin{tabular}{|c|c|c|c|c|c|c|}
\hline \multirow[b]{2}{*}{ PLM Unit } & \multicolumn{2}{|c|}{ Cold case $T[K]$} & \multicolumn{2}{|c|}{ Hot case $T[K]$} & \multirow[b]{2}{*}{$\begin{array}{l}\text { Req } \\
{[K]}\end{array}$} & \multirow{2}{*}{$\underset{[K]}{\operatorname{Margin}}$} \\
\hline & $\begin{array}{c}\text { JT diff node } \\
(37.5 \mathrm{~mW})\end{array}$ & $\begin{array}{c}\text { JT boundary } \\
\text { @ 28K }\end{array}$ & $\begin{array}{c}\text { JT diff node } \\
(37.5 \mathrm{~mW})\end{array}$ & $\begin{array}{c}\text { JT boundary } \\
\text { @ 28K }\end{array}$ & & \\
\hline $\begin{array}{l}\text { SVM top plate (fixed } \\
\text { boundary temperature) }\end{array}$ & \multicolumn{2}{|c|}{270.00} & \multicolumn{2}{|c|}{290.00} & - & - \\
\hline $\begin{array}{l}\text { SVM Radiative shield (fixed } \\
\text { boundary temperature) }\end{array}$ & \multicolumn{2}{|c|}{180.00} & \multicolumn{2}{|c|}{200.00} & - & - \\
\hline
\end{tabular}




\begin{tabular}{|l|c|c|c|c|c|c|}
\hline VG1 & 142.4 & 142.4 & 151.5 & 151.5 & 180 & 10 \\
\hline VG2 & 92.5 & 92.5 & 97.3 & 97.3 & 120 & 10 \\
\hline VG3 & 55.7 & 55.7 & 57.0 & 56.9 & 70 & 10 \\
\hline Telescope M1 & 49.9 & 49.8 & 50.7 & 50.6 & 70 & 10 \\
\hline Telescope M2 & 49.6 & 49.5 & 50.5 & 50.4 & 70 & 10 \\
\hline Telescope Baffle & 48.9 & 48.8 & 49.7 & 49.6 & 60 & 10 \\
\hline TOB & 50.0 & 49.9 & 50.9 & 50.8 & 60 & 10 \\
\hline FGS/NIRP CFEEs & 50.5 & 50.4 & 51.4 & 51.3 & 70 & 10 \\
\hline FGS/NIRP detectors & 49.7 & 49.6 & 50.5 & 50.4 & 70 & 10 \\
\hline Instrument radiator & 49.4 & 49.3 & 50.2 & 50.1 & 60 & 10 \\
\hline AIRS CFEEs & 50.6 & 50.5 & 51.4 & 51.3 & 62 & 10 \\
\hline AIRS detectors & 36.7 & 28.2 & 37.5 & 28.2 & 42 & 5 \\
\hline JT cold end & 36.5 & 28.0 & 37.4 & 28.0 & 40 & 5 \\
\hline
\end{tabular}

The unit temperatures maps for all cases are graphically shown in the next figures, comparing the Cold and Hot cases: general views of the PLM are compared in Figure 7, while Figure 8 and Figure 9 show the details of the coldest units in both cases.
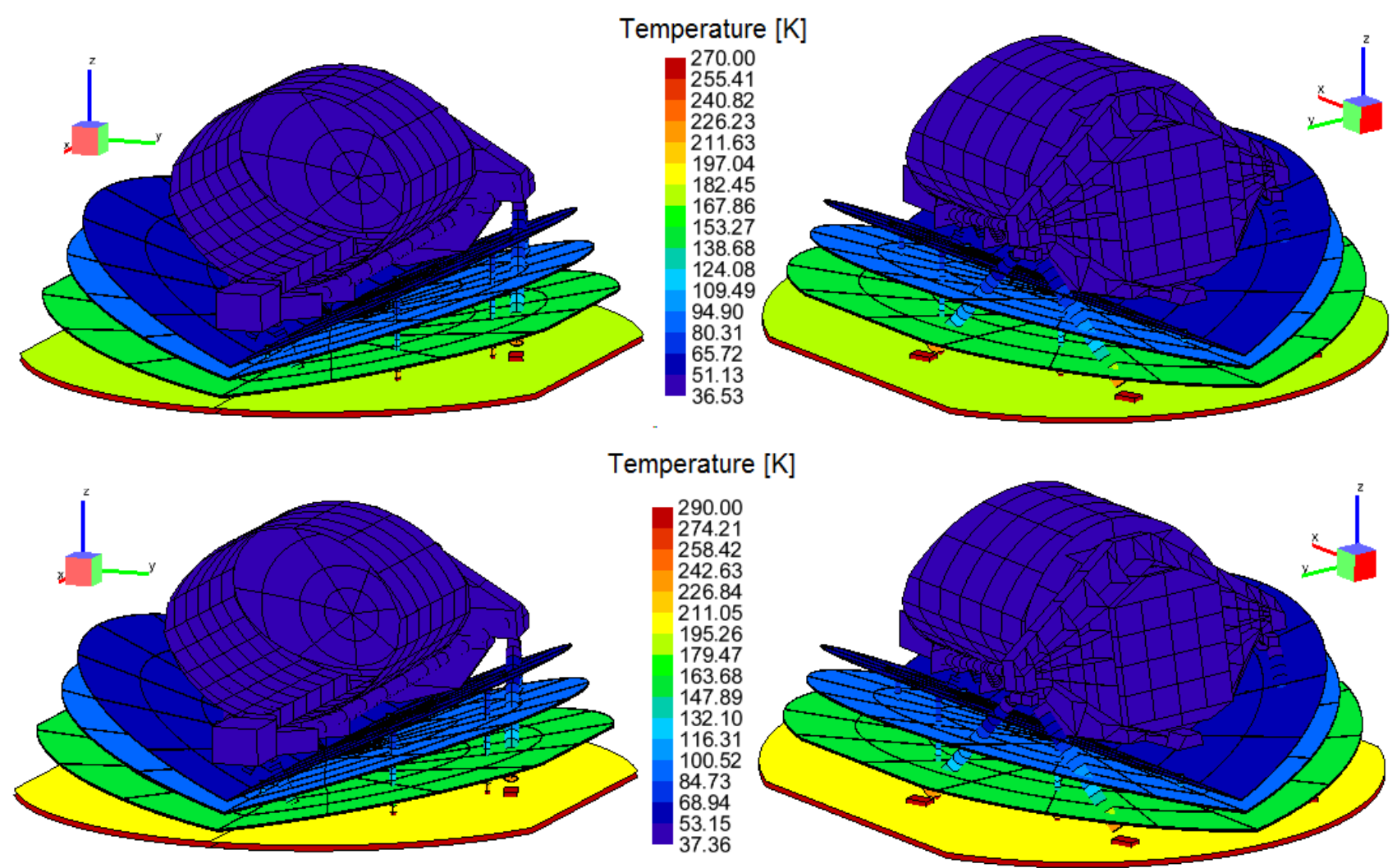

Figure 7: Cold Case (upper panel) and Hot Case (lower panel) steady-state results in nominal conditions, general views of the PLM model. 

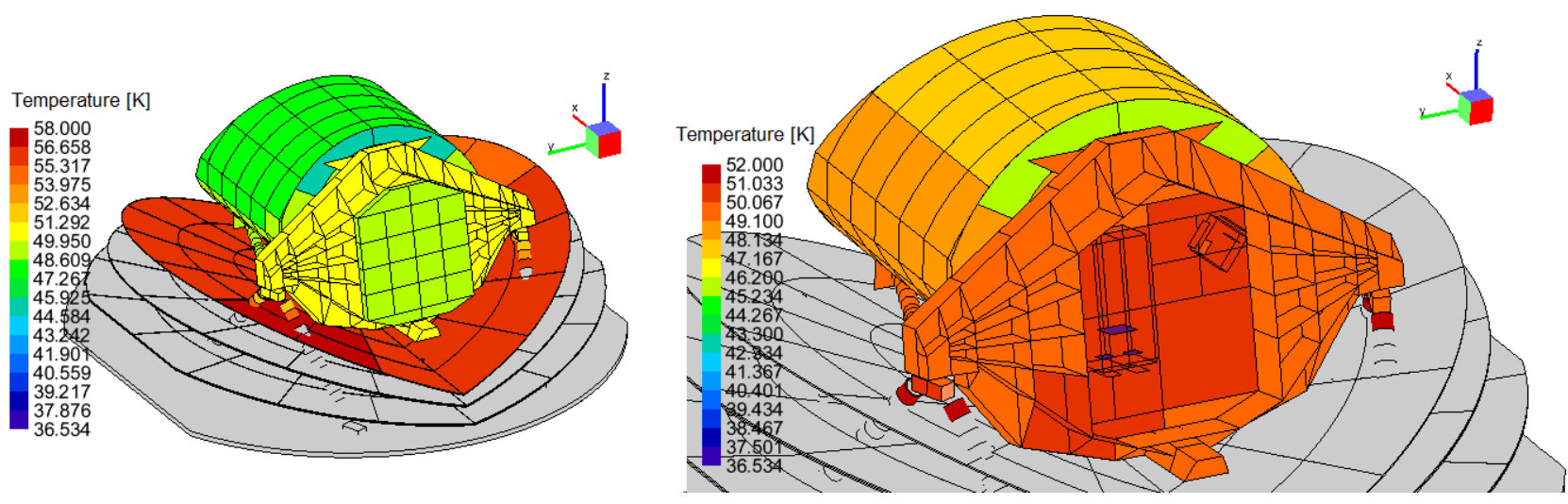

Figure 8: Cold case steady-state results in nominal conditions, detailed views of the cold PLM. Left view shows the radiator, OB and baffle temperatures. Right side: the module boxes are hidden in transparency to show AIRS and FGS/NIRP CFEEs, detectors and the JT cold end.

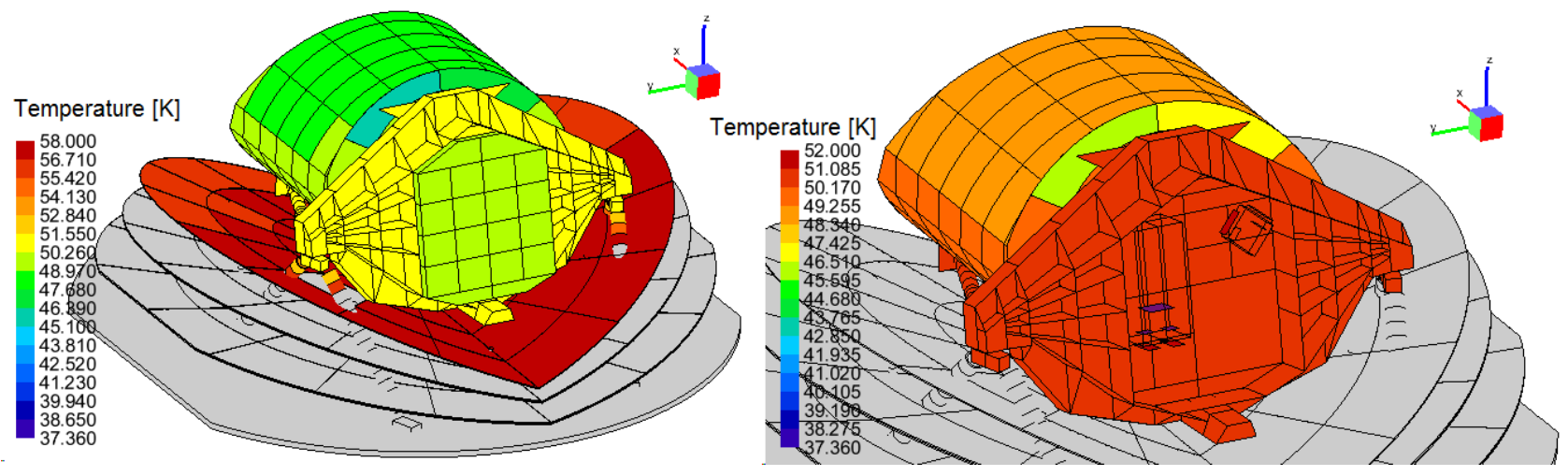

Figure 9: Hot case steady-state results in nominal conditions, detailed views of the cold PLM. Left view shows the radiator, OB and baffle temperatures. Right side: the module boxes are transparent to show AIRS and FGS/NIRP CFEEs, detectors and the JT cold end.

Figure 10 reports a detailed view of the Telescope Assembly in the Cold Case, to show the thermal uniformity reached by the passive cooling. The M1 thermal gradient is lower than few $\mathrm{mK}$.

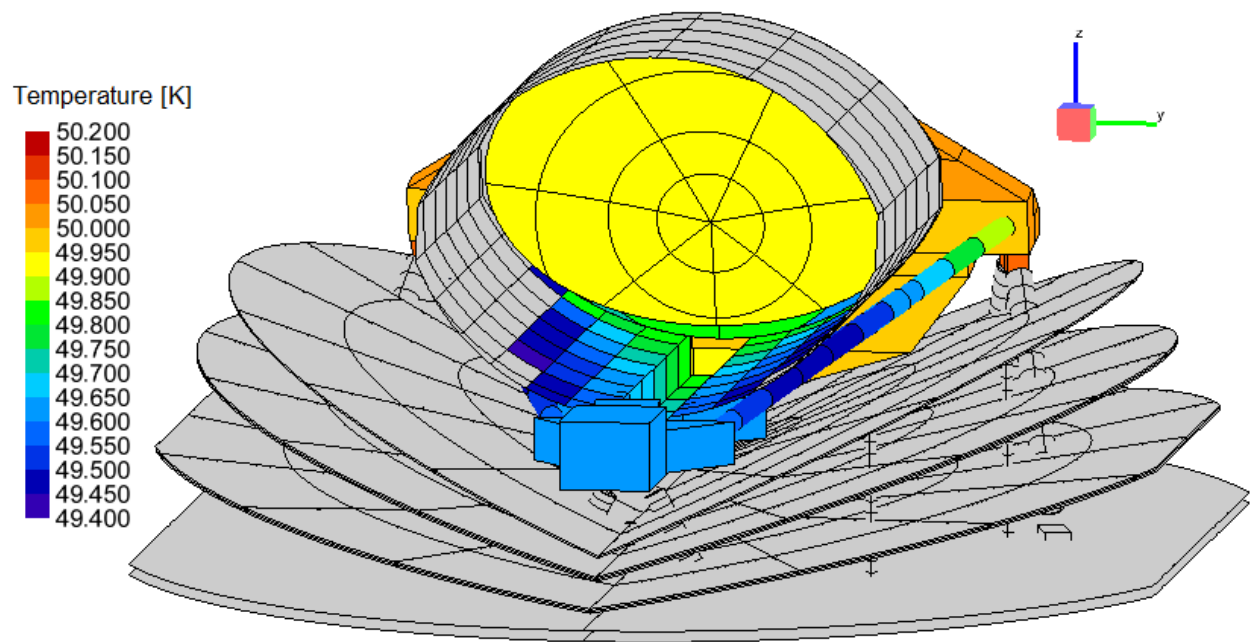

Figure 10: Telescope Assembly nodes temperature in the Cold Case 
The G/TMM performances in terms of heat fluxes to/from the PLM units and rejection capabilities of the passive cooling radiators are summarized in the following table. The loads to the thermal interfaces are evaluated by balancing the input/output heat fluxes to/from each node through all conductors.

Table 4: Heat exchange at the main external/internal interfaces and between units for Cold and Hot cases in nominal conditions.

\begin{tabular}{|c|c|c|c|c|c|c|}
\hline Interface & Location & $\underset{[K]}{\operatorname{Req} T}$ & $\begin{array}{c}\text { Cold Case T } \\
{[\mathrm{K}]}\end{array}$ & $\begin{array}{l}\text { Cold Case } \\
\text { Load }[\mathrm{mW}]\end{array}$ & $\begin{array}{c}\text { Hot Case T } \\
{[\mathrm{K}]}\end{array}$ & $\begin{array}{c}\text { Hot Case Load } \\
{[\mathrm{mW}]}\end{array}$ \\
\hline \multirow{2}{*}{$\begin{array}{c}\text { TIFO } \\
\text { (PLM to SVM) }\end{array}$} & SVM Conducted & $\leq 290$ & 270 & -6040 & 290 & -8720 \\
\hline & SVM Radiative & $\leq 290$ & 180 & -1624 & 200 & -2733 \\
\hline TIF1 & V-Groove 1 & $\leq 200$ & 142.4 & 7338 & 151.5 & 9351 \\
\hline TIF2 & V-Groove 2 & $\leq 120$ & 92.5 & 1238 & 97.3 & 1501 \\
\hline TIF3 & V-Groove 3 & $\leq 70$ & 55.7 & 1345 & 57.0 & 1481 \\
\hline TIF4 & TOB/Baffle & $\leq 60$ & 50.0 & 706 & 50.9 & 753 \\
\hline TIF5 & $\begin{array}{l}\text { Instrument } \\
\text { Radiator }\end{array}$ & $\leq 60$ & 49.4 & 102 & 50.2 & 106 \\
\hline TIF6 & JT cold end & $<40$ & $\begin{array}{l}36.5^{1} \\
28.0^{2}\end{array}$ & $\begin{array}{l}37.5^{1} \\
48.4^{2}\end{array}$ & $\begin{array}{l}37.4^{1} \\
28.0^{2}\end{array}$ & $\begin{array}{l}37.5^{1} \\
49.5^{2}\end{array}$ \\
\hline
\end{tabular}

When the JT cold end is modelled as a diffusion node the heat balance is always $37.5 \mathrm{~mW}$ because this is a fixed boundary condition. The parameter that shows the heat balance performances of the JT heat exchanger is the equilibrium temperature of the cold end (and of the AIRS detectors). This temperature ranges from 36.5 to $37.5 \mathrm{~K}$ for the Cold and Hot Cases. The AIRS detectors temperature is $0.2 \mathrm{~K}$ higher than the cold end.

In the case of the JT boundary at $28 \mathrm{~K}$ (Table 4) it is possible to evaluate the heat flow to the cold end node. The load to the boundary is $48.4 \mathrm{~mW}$ in the Cold Case and $49.5 \mathrm{~mW}$ in the Hot Case. These numbers are marginal to the baseline heat lift expected from the cooler but they correspond to detector temperatures well below the requirement ( $42 \mathrm{~K})$. In this case a significant margin on the heat lift could be achieved by relaxing the resulting temperature of the AIRS detectors. Moreover the dissipation of the AIRS detectors used in the TMM, already includes a margin of $35 \%$ due to an overestimation of the parasitic leaks, that are the dominant contribution of the SCA's heat balance.

The tables above provide an indication of the efficiency and performances of the ARIEL PLM passive design. The VGroove 3 is capable of rejecting more than $1 \mathrm{~W}$ at a temperature of $55 \mathrm{~K}$ ( $15 \mathrm{~K}$ below the requirement), while the telescope baffle and the instrument radiator dissipate to space, respectively, $0.7 \mathrm{~W}$ and $0.1 \mathrm{~W}$ at temperatures below the requirements.

\section{CONCLUSIONS}

We presented the baseline thermal architecture of the payload for the ARIEL mission at the time of the Mission Selection Review. From the basic thermal requirements of the instrument the whole design has been derived and the thermal interfaces to the Spacecraft have been identified. The general thermo-mechanical configuration of the units and the thermal interfaces has been described. The expected performances of the thermal control configuration have been analyzed on the basis of simulations in steady state and transient conditions. The results indicate that the present design is compliant to the instrument and interface requirements for temperature control and heat fluxes to the cooling stages. The G/TMM output demonstrates that the ARIEL PLM thermal architecture and passive cooling design are really efficient in rejecting heat, taking full advantage of the L2 favourable thermal conditions. The Telescope Optical Bench, 
the Telescope Baffle and the Instrument Cold Radiator work together as a single large surface, representing the coldest radiative stages in the spacecraft, at a temperature lower than the last V-Groove.

In Figure 11 the operating temperature ranges of the Cold to Hot Cases of the main PLM units for each JT cold end simulation case are reported as grey boxes. These boxes are shown in the picture together with the requirements (blue bars) plus the margins (yellow bars) required by the present level of the design.

For both thermal cases, all temperatures result below, or at, the required values including margin, indicating a fully compliant thermal architecture with some extra space for further optimizations. In phase B1 the thermal architecture will be optimized to reduce the uncertainty margins and increase the design margins on the passive and active cooling stages, especially the coldest ones.
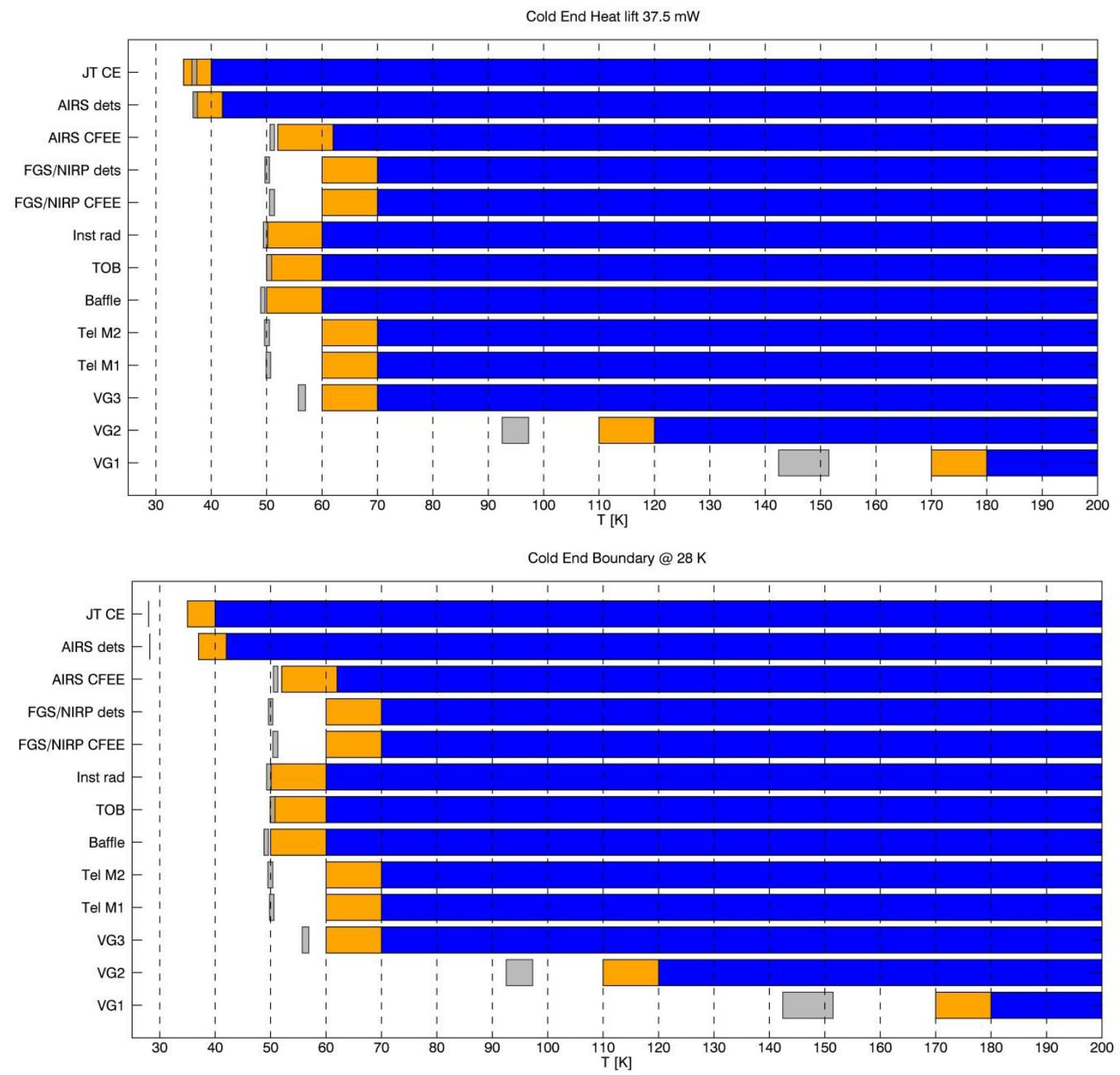

Figure 11: The temperature ranges (grey bars) resulting in Cold and Hot case for the main PLM units are compared to the requirement (blue bars) with margins (yellow bars). Top graph reports the values for the JT cold end with fixed heat lift (37.5 $\mathrm{mW}$ ); the bottom picture shows the same values in the case of a JT cold end as a boundary node at $28 \mathrm{~K}$.

The ARIEL PLM loads, especially for what concerns detectors dissipation, mechanical supports, piping and harness leaks, have been evaluated on the basis of the present knowledge of the units performances and of the heritage from more advanced projects (MIRI, Planck, Herschel, Euclid etc.). Conservative estimations have been assumed and verified by 
thermal analysis with the PLM G/TMM. The budget resulting at the external and internal thermal interfaces is reported in Table 4. Even in the worst case of boundary conditions and parasitic leaks the total load on the JT cold end is well below the maximum estimated heat lift capacity of the cooler $(200 \mathrm{~mW})$. If this numbers will be confirmed in the next design steps, it will be possible to relax the heat load requirement to the cold end heat exchangers and optimize the cryochain performance in a narrower range. The fact that the heat fluxes reported in that Table correspond to interface temperatures lower than required, indicate that extra margin is available for optimizations in the next phase.

The thermal architecture here described provides a solid basis for the future design evolution of the PLM thermal architecture.

\section{ACKNOWLEDGEMENTS}

We want to acknowledge here the fundamental contribution of the ARIEL System Team to this work. The Italian participation to the ARIEL mission was supported by the Italian Space Agency (ASI) in the framework of the ASI-INAF contract n. 2015-038-R.0.

\section{REFERENCES}

[1] Tinetti, G., et al., "The science of ARIEL (Atmospheric Remote-sensing Infrared Exoplanet Large-survey)", Space Telescopes and Instrumentation 2016: Optical, Infrared, and Millimeter Wave, Proc. of SPIE Vol. 9904, 99041X (2016) doi: 10.1117/12.2232370

[2] Eccleston, P., et al, "An integrated payload design for the Atmospheric Remote-sensing Infrared Exoplanet Large-survey (ARIEL)”, Space Telescopes and Instrumentation 2016: Optical, Infrared, and Millimeter Wave, Proc. of SPIE Vol. 9904, 990433 (2016) doi: 10.1117/12.2232878

[3] ARIEL Assessment Study Report (Yellow Book), ESA/SCI(2017)2, (2017)

[4] Planck collaboration, "Planck early results. II. The thermal performance of Planck", Astronomy \& Astrophysics, 536, A2 (2011)

[5] Morgante, G., Terenzi, L., Eccleston, P. et al., "Thermal control system of the Exoplanets Characterisation Observatory payload: design and predictions", Experimental Astronomy (2015) 40: 771. https://doi.org/10.1007/s10686-015-9469- (2015)

[6] Focardi, M., Pace, E., Farina, M. et al., "The ARIEL Instrument Control Unit Design", Experimental Astronomy (2017) https://doi.org/10.1007/s10686-017-9560-3

[7] V. Da Deppo, K. Middleton, M. Focardi, G. Morgante, E. Pace, R. Claudi, G. Micela, "An afocal telescope configuration for the ESA Ariel mission", Proc. SPIE 10562, International Conference on Space Optics - ICSO 2016, 105624W (2017) doi: 10.1117/12.2296108 